\title{
P04-24. Frequencies of HIV Env-specific B cells and neutralizing antibodies during DNA-prime/protein-boost immunization with three different Env immunogens*
} P Tawde ${ }^{* 1}$, K Strouss ${ }^{1}$, Z Kraft ${ }^{1}$, M Gauduin ${ }^{2}$ and L Stamatatos ${ }^{1}$

\author{
Address: ${ }^{1}$ HIV Viral Vaccine Program, Seattle Biomedical Research Institute (SBRI), Seattle, WA, USA and ${ }^{2}$ Southwest Foundation for Biomedical \\ Research, San Antonio, TX, USA \\ * Corresponding author
}

from AIDS Vaccine 2009

Paris, France. 19-22 October 2009

Published: 22 October 2009

Retrovirology 2009, 6(Suppl 3):P52 doi:10.1186/1742-4690-6-S3-P52

This abstract is available from: http://www.retrovirology.com/content/6/S3/P52

C 2009 Tawde et al; licensee BioMed Central Ltd.

\section{Background}

To address the challenging goal of eliciting broadly neutralizing antibodies (NAbs) to HIV, several immunization strategies have been employed so far with little success. We are interested in identifying immunization strategies that will aid in more robust generation of NAbs both in titer and sustainability. This study investigates the relationship between the frequencies of anti-HIV-1 Env B cells generated during DNA-prime protein-boost immunization with the titer of anti-Env antibodies, using three different Env immunogens.

\section{Methods}

Rhesus macaques were immunized with either soluble trimeric wild type gp140 (wt-gp140), or monomeric gp120 (m-gp120), or CD4-binding site mutant (D368R) gp140 (D368R-gp140). Throughout immunization, the frequency of HIV Env-specific B cells was evaluated by multicolor flow cytometry, anti-Env antibody titers by ELISA, and neutralization activity assayed using TZM-bl assay.

\section{Results}

The frequency of HIV Env specific plasmablast subset (CD3- CD20+ CD27high CD38 high) and titers of antiEnv antibodies increased as a result of protein boost in comparison with DNA prime in all immunized animals. The average frequency of Env specific B cells after protein immunization was $0.72 \%(0.47-0.99 \%)$ for the wt- gp $140,0.44 \%(0.35-0.52 \%)$ for the gp 120 , and $0.35 \%$ $(0.13-0.56 \%)$ for the D368R gp140 immunized animals. However, no significant correlations can be drawn between the titer of Env specific antibodies and frequency of Env specific B cells in any group. The wt-gp140 was a more effective immunogen than m-gp120 in eliciting weak cross-NAbs. Interestingly, the wt-gp140 was also more effective in eliciting cross-NAbs than the D368Rgp140; potentially suggesting that the development of cross-NAbs by wt-gp140 depends on the structural integrity of the CD4-binding site.

\section{Conclusion}

Protein boosting is required for the development of high frequencies of anti-HIV Env antibodies, and ability of trimeric gp140 to elicit cross-NAbs will depend on the structural integrity of the CD4-binding site.

\section{Acknowledgement}

* Research was supported by National Institutes of Health grant number R01 AI047708-11. 